\title{
Serological Screening of Celiac Disease in Patients with Juvenile Idiopathic Arthritis
}

\author{
Payman Sadeghi, MD,2; Kobra Salari, MD; Vahid Ziaee, MD $^{1,2}$; Nima Rezaei, MD $^{3}$; Kambiz Eftekhari, MD ${ }^{4,5 *}$ \\ ${ }^{1}$ Children's Medical Center, Pediatrics Center of Excellence, Tehran University of Medical Sciences, Tehran, Iran \\ ${ }^{2}$ Pediatric Rheumatology Iranian Society \\ ${ }^{3}$ Research Center for Immunodeficiencies, Children's Medical Center, Tehran University of Medical Sciences, Tehran, Iran \\ ${ }^{4}$ Pediatric Gastroenterology and Hepatology Research Center, Children's Medical Center, Tehran University of Medical Sciences, \\ Tehran, Iran \\ ${ }^{5}$ Department of Pediatrics, Bahrami Children's Hospital, Tehran University of Medical Sciences, Tehran, Iran
}

\begin{abstract}
Background: There is a possible association between celiac disease (CD) and juvenile idiopathic arthritis (JIA). Our aim was to evaluate the serological incidence of CD in patients with JIA. Children under 16 years of age with JIA who did not respond adequately to routine treatment, who referred to the pediatric centers of Tehran University of Medical Sciences (2017-2019), were enrolled in this study. Manifestations of CD were also evaluated. CD-related serological screening tests were measured. Seventyeight patients were enrolled in the study. Their mean age was $7.9 \pm 3.9$ (1.6-16) years. Three patients with oligoarticular JIA had Anti-TTG-Ab levels above normal (prevalence $=3.8 \%$ ). None of them had symptoms of CD. There were no significant statistical differences in terms of growth disorders, sex distribution, and different subtypes of JIA ( $P$ value $>0.05)$ between the groups (seropositive vs. sero-negative). In one case, $\mathrm{CD}$ was confirmed by pathology and the gluten-free diet was recommended. The absence of $\mathrm{CD}$ symptoms in patients with JIA does not rule out concomitant CD.

Keywords: Anti-tissue transglutaminase antibody, Celiac disease, Children, Juvenile Idiopathic arthritis

Cite this article as: Sadeghi P, Salari K, Ziaee V, Rezaei N, Eftekhari K. Serological screening of celiac disease in patients with juvenile idiopathic arthritis. Arch Iran Med. 2021;24(10):783-785. doi: 10.34172/aim.2021.116
\end{abstract}

Received: March 18, 2020, Accepted: January 24, 2021, ePublished: October 1, 2021

\section{Introduction}

Juvenile idiopathic arthritis (JIA), the most common cause of non-infectious chronic arthritis in children younger than 16 years, is occasionally associated with extraarticular symptoms, such as growth retardation (weight or height) and gastrointestinal manifestations. ${ }^{1}$

Celiac disease (CD) can cause gastrointestinal and joint involvement. Therefore, CD should be considered in patients with arthritis associated with failure to thrive (FTT) and gastrointestinal symptoms. ${ }^{1} \mathrm{CD}$ is a gluten-sensitive enteropathy which leads to autoimmune reactions and production of autoantibodies in genetically susceptible individuals. ${ }^{2}$ The association between CD and JIA has been reported in some studies and its prevalence is reported at $1 \%-10 \% .^{3}$ Therefore, CD should be considered in patients with JIA when unexplained extraarticular symptoms occur. ${ }^{3}$ There are still some questions about the association of these diseases; for example, whether CD should be screened in all patients with JIA before initiation of treatment. Is this association due to the common background of two different diseases with different treatments ${ }^{3,4}$ ? The results of different studies are not similar and the relationship between these two diseases is currently unclear. In Iran, only one study was conducted on children and adults with JIA and it did not show a higher incidence of CD in these patients. ${ }^{5}$ In another study, the prevalence of CD was higher in adults with JIA. ${ }^{6}$

Our aim was to screen CD in patients with JIA at the pediatric rheumatology centers of Tehran University of Medical Sciences.

\section{Materials and Methods}

This was a cross-sectional study performed in children with JIA from July 2017 to March 2019 at the Pediatric Rheumatology Centers of Tehran University of Medical Sciences. The patients were classified into different subgroups according to the ILAR- JIA criteria.

Inclusion criteria were the following: children under the age of 16 with JIA, whose disease did not respond appropriately to treatment (resistant after one year of treatment) or relapsed after undergoing remission.

Exclusion criteria were the following: known immune deficiencies, renal failure, liver failure, and known malabsorption syndromes such as CD or inflammatory bowel disease.

Seventy-eight patients were enrolled after obtaining informed consent from them or their parents. All data were recorded in a checklist which included demographic information (age, gender), clinical information such as 
type of the disease, duration of disease, type of medication used, and control degree of disease (according to clinical and laboratory findings). Also, the growth status, presence and type of gastrointestinal symptoms, and laboratory findings were recorded. Weight and height were measured and compared with standard growth charts of WHO and CDC. Growth classification was done as follows: growth retardation or FTT [type I (weight retardation), type II (height and weight retardation)], and obesity.

Then $5 \mathrm{~mL}$ venous blood samples were taken and analyzed for Anti-tissue transglutaminase antibodies (IgA) (Anti TTG-IgA) by means of enzyme-linked immunosorbent assay (ELISA). Anti TTG Antibody levels of $20(\mathrm{U} / \mathrm{mL})$ and above were considered positive according to the kit manufacturer's reference. Cases with Anti TTG antibody levels ranging from 5 to 20 (U/ $\mathrm{mL}$ ) were considered suspicious and re-measured. Since selective immunoglobulin A deficiency is more common in the celiac patients, total immunoglobulin A levels (Total IgA) were measured by nephelometry method, to confirm the true negative results of the Anti-TTG antibody. The cutoff point of this test was considered to be less than 33 $\mathrm{mg} / \mathrm{dL}$ according to the kit manufacturer's reference. If the primary serologic results were abnormal, the patients were referred to a pediatric gastroenterologist for the definite diagnosis of CD (by upper GI endoscopy and biopsy). The samples were classified on the basis of "March criteria" by a pathologist. Finally, the diagnosis of CD was confirmed or rejected by pediatric gastroenterologists. In case of low total serum IgA, the patient was referred to a pediatric immunologist for further immune deficiency evaluations.

\section{Data Analysis}

The data were analyzed using SPSS version 25. The descriptive data were reported by descriptive statistics (median, standard deviation, frequency, relative frequency). For comparison of qualitative variables between two groups, the chi-square test (Fisher's exact test) was done and for quantitative variables, the student sample $t$ test in case of normal distribution and MannWhitney test in case of abnormal distribution were done. $P$-values less than 0.05 were considered statistically significant.

\section{Results}

The ratio of girls to boys was $2 / 1$ and the mean age was $7.9 \pm 3.9$ years $(1.6-16$ years $)$, the age at disease onset was $5 \pm 2.3(0.3-12)$ years, and duration of the disease was $33 \pm 34$ (1-144) months. In these patients, $32 \%$ had growth retardation [type $1(14.1 \%, \mathrm{n}=11)$ and type 2 $(17.9 \%, \mathrm{n}=14)$ ] and $6.4 \%$ were overweight. Twentyfive patients $(32 \%)$ had gastrointestinal symptoms including anorexia, abdominal pain, constipation, and flatulence in decreasing order of frequency. None of the patients had low levels of total serum IgA. Seven patients had anti-TTG Ab (IgA) levels above 5 in the primary screening which was considered suspicious according the laboratory kit. However, after repeating the test, only three patients $(8.3 \%)$ had antibody levels above 20 which were considered positive. The antibody level in two patients was higher than 200 and the third patient had $133 \mathrm{IU} /$ $\mathrm{mL}$ (in this patient, the primary screening test was 7). The mean age in these sero-positive children was 6.7 and the mean age of onset of disease was 5 years. The clinical and laboratory characteristic of the positive serologic tests are summarized in Table 1. All had a normal growth pattern with no gastrointestinal complaints.

There were no significant statistical differences in terms of growth disorders $[P$ value $=1.00,95 \%$ CI: $1.0(1.00-$ $1.00)]$, sex distribution $(P$ value $=0.700)$, and different subtypes of JIA $[P$ value $=0.620,95 \%$ CI: $0.6(0.60-0.63)]$ between the two groups of positive and negative antiTTG. A gluten-free diet was started in the patient with a definitive diagnosis of $\mathrm{CD}$ based on the pathology result (March III $_{b}$ ). After two months of diet, all clinical (including musculoskeletal) manifestations subsided completely. The drugs were tapered gradually and then discontinued. The patient had no recurrence after 12 months, continues on gluten-free diet and has been monitored by the Anti-TTG level.

\section{Discussion}

The aim of this study was serological screening of JIA patients for CD. Based on the findings, the prevalence of high serum Anti TTG Antibody is $8.3 \%$. The prevalence of $\mathrm{CD}$ in healthy asymptomatic children has been reported at $0.5-2 \% .^{7,8}$ In our study, the mean age was lower than those studies. The prevalence of CD was higher in our study and the sex dominancy was female/male ratio of $2 / 1$, in contrast to Farahmand and colleagues' study. ${ }^{7}$ Concerning the similarities of lab kits, geographic region and the population between our study and Farahmand and colleagues' study, this difference could be valuable.

The prevalence of CD among JIA patients was reported differently among several studies (1.8\%-6.6\%). ${ }^{5,9}$ The reason for the false-positive result of Anti-TTG IgA could be the presence of some antibodies due to other conditions or to increased polyclonal IgA levels. ${ }^{9,10}$ Sometimes, in spite

Table 1. Clinical and Laboratory Characteristics of Anti-TTG Positive Patients

\begin{tabular}{ccccccc}
\hline Age $(\mathbf{y})$ & Gender & Type of Disease & Duration of Illness (mon) & Gl Symptoms Growth Disorder & Anti TTG IgA & Intestinal Biopsy Result \\
\hline 10 & Male & Oligoarticular (knee) & 27 & No & March III & No \\
7.5 & Female & Oligoarticular (knee) & 50 & No & $>200$ \\
6 & Male & Oligoarticular (knee) & 13 & 130 & Not done \\
\hline
\end{tabular}

Gl, gastrointestinal; Anti-TTG IgA, anti-tissue transglutaminase antibody. 
of a positive serologic result for $\mathrm{CD}$, the inflammation of small bowel mucosa could be improved by corticosteroids or immunosuppressive drugs. In such cases, despite a biopsy, a definitive diagnosis can be very challenging especially in JIA patients under treatment. ${ }^{11}$ Selective IgA deficiency and use of immunosuppressive drugs could be important reasons for false-negative results of the AntiTTG test. ${ }^{2}$ In these situations, measuring endomysial antibody is useful, and if there is a high suspicion, biopsy is recommended. One negative result of anti-TTG Ab IgA cannot definitively rule out the disease (a CD with negative Anti-TTG Ab is called sero-negative CD). ${ }^{2}$ On the basis of multiple studies including ours, absence of clinical manifestation or growth failure in JIA patients cannot exclude CD. ${ }^{3,5}$ Therefore, patients with rheumatological complaints are considered to be at higher risk for CD. Also, the presence of symptoms such as inadequate response to routine treatments of JIA can raise the suspicion of the CD. In different studies, a gluten-free diet could reduce joint involvement, ${ }^{9,12}$ musculoskeletal complaints, ${ }^{4}$ and improve growth rate. ${ }^{9}$ In our study, this method helped to discontinue the drug therapy.

\section{Study Limitations}

Among the limitations of our study are the following: lack of consent of some patients, unwillingness of some positive serologic patients to undergo endoscopy regarding lack of obvious gastrointestinal complaints or aggressive nature of the medical procedure, the patient's unwillingness to repeat the test.

In conclusion, $\mathrm{CD}$ can be asymptomatic and only be diagnosed by screening. In JIA patients with concomitant growth disorders or even failure to respond to appropriate treatment for underlying disease, screening for concomitant CD is necessary.

\section{Authors' Contribution}

PS, KS, VZ, NR, and KE designed the study. PS, KS, and KE were responsible for acquisition of data and wrote the paper. PS, VZ, NR, and KE were analysis and interpretation of data. PS, KS, VZ, NR, and KE were drafting of the manuscript. PS, VZ, and KE were Critical revised of the manuscript for important intellectual content. PS, KS, VZ, NR, and KE were study supervision. All authors read and approved the final version to be published.

\section{Ethical Statement}

The study was approved by the Ethics Committee of Tehran University of Medical Sciences (ethics number: IR.TUMS.MEDICINE. REC.1396.4887). The research objectives and procedures were explained to the parents and children; additionally, they were assured of the confidentiality of their information. Moreover, informed consent was obtained from parents, and they were all assured that they could leave the study at any time.

\section{Conflict of Interest Disclosures}

The authors reported that they have no conflict of interest.

\section{Acknowledgment}

We thank all the patients and their parents for having allowed us to perform this study. Also, we appreciate the cooperation of Dr. Diana Diaz in writing this article.

\section{References}

1. Wu EY, Bryan AR, Rabinovich CE. Juvenile Idiopathic Arthritis (Chapter 155). In: Richard EB, Robert MK, Bonita FS, Joseph W SGI, Nina FS, eds. Nelson Text Book of Pediatrics. 20th ed. Philadelphia: Saunders Elsevier; 2016. p. 1164-7.

2. Lebwohl B, Sanders DS, Green PHR. Coeliac disease. Lancet. 2018;391(10115):70-81. doi: 10.1016/s01406736(17)31796-8.

3. Sherman $Y$, Karanicolas R, DiMarco B, Pan N, Adams $A B$, Barinstein LV, et al. Unrecognized celiac disease in children presenting for rheumatology evaluation. Pediatrics. 2015;136(1):e68-75. doi: 10.1542/peds.2014-2379.

4. Sur LM, Floca E, Sur G, Rednic S. Serological and genetic evidence of celiac disease in juvenile arthritis and rheumatoid arthritis. Int J Celiac Dis. 2016;4(3):82-3. doi: 10.12691/ijcd4-3-8.

5. Moghtaderi M, Farjadian S, Aflaki E, Honar N, Alyasin S, Babaei M. Screening of patients with juvenile idiopathic arthritis and those with rheumatoid arthritis for celiac disease in southwestern Iran. Turk J Gastroenterol. 2016;27(6):521-4. doi: 10.5152/tjg.2016.16354.

6. Elhami E, Zakeri Z, Sadeghi A, Rostami-Nejad M, Volta U, Zali MR. Prevalence of celiac disease in Iranian patients with rheumatologic disorders. Gastroenterol Hepatol Bed Bench. 2018;11(3):239-43.

7. Farahmand F, Mir-Nasseri MM, Shahraki T, Yourdkhani F, Ghotb S, Modaresi V, et al. Prevalence of occult celiac disease in healthy Iranian school age children. Arch Iran Med. 2012;15(6):342-5.

8. Ahadi Z, Shafiee G, Razmandeh R, Keshtkar AA, Najafi Sani $M$, Azemati $B$, et al. Prevalence of celiac disease among the Iranian population: a systematic review and meta-analysis of observational studies. Turk J Gastroenterol. 2016;27(2):122-8. doi: 10.5152/tjg.2015.150191.

9. Al-Mayouf SM, Al-Mehaidib Al, Alkaff MA. The significance of elevated serologic markers of celiac disease in children with juvenile rheumatoid arthritis. Saudi J Gastroenterol. 2003;9(2):75-8.

10. Stoll ML, Patel AS, Christadoss ML, Punaro M, Olsen NJ. IgA transglutaminase levels in children with juvenile idiopathic arthritis. Ann Paediatr Rheumatol. 2012;1(1):31-5. doi: 10.5455/apr.112220111551.

11. Rostom A, Murray JA, Kagnoff MF. American Gastroenterological Association (AGA) Institute technical review on the diagnosis and management of celiac disease. Gastroenterology. 2006;131(6):1981-2002. doi: 10.1053/j. gastro.2006.10.004.

12. Garg K, Agarwal P, Gupta RK, Sitaraman S. Joint involvement in children with celiac disease. Indian Pediatr. 2017;54(11):9468. doi: 10.1007/s13312-017-1188-x. 